\title{
Counterexample to Strong Diamagnetism for the Magnetic Robin Laplacian
}

\section{Ayman Kachmar ${ }^{1} \cdot$ Mikael P. Sundqvist $^{2}$}

Received: 20 December 2019 / Accepted: 13 May 2020 / Published online: 5 July 2020

(C) The Author(s) 2020

\section{Abstract}

We determine a counterexample to strong diamagnetism for the Laplace operator in the unit disc with a uniform magnetic field and Robin boundary condition. The example follows from the accurate asymptotics of the lowest eigenvalue when the Robin parameter tends to $-\infty$.

Keywords Magnetic Laplacian · Robin boundary condition · Eigenvalues · Diamagnetic inequalities

Mathematics Subject Classification (2010) Primary 35P15 · 47A10 · 47F05

\section{Introduction}

\subsection{Magnetic Robin Laplacian}

We denote by $\Omega=\left\{x \in \mathbb{R}^{2}:|x|<1\right\}$ the open unit disc and by $\Gamma=\partial \Omega=\{x \in$ $\left.\mathbb{R}^{2}:|x|=1\right\}$ its boundary. We study the lowest eigenvalue of the magnetic Robin Laplacian in $L^{2}(\Omega)$,

with domain

$$
\mathcal{P}_{\gamma}^{b}=-\left(\nabla-i b \mathbf{A}_{0}\right)^{2}
$$

$$
D\left(\mathcal{P}_{\gamma}^{b}\right)=\left\{u \in H^{2}(\Omega): v \cdot\left(\nabla u-i b \mathbf{A}_{0}\right) u+\gamma u=0 \quad \text { on } \partial \Omega\right\} .
$$

Mikael P. Sundqvist

mikael.persson_sundqvist@math.lth.se

Ayman Kachmar

ayman.kashmar@gmail.com

1 Department of Mathematics, Lebanese University, Nabatieh, Lebanon

2 Department of Mathematical Sciences, Lund University, Box 118, 22100 Lund, Sweden 
Here $v$ is the unit outward normal vector of $\Gamma, \gamma<0$ the Robin parameter and $b>0$ is the intensity of the applied magnetic field. The vector field $\mathbf{A}_{0}$ generates the unit magnetic field and is defined as follows

$$
\mathbf{A}_{0}\left(x_{1}, x_{2}\right)=\frac{1}{2}\left(-x_{2}, x_{1}\right) .
$$

To be more precise, the operator $\mathcal{P}_{\gamma}^{b}$ is defined as the Friedrichs extension, starting from the quadratic form [8, Ch. 4],

$$
H^{1}(\Omega) \ni u \mapsto \mathcal{Q}_{\gamma}^{b}(u):=\int_{\Omega}\left|\left(\nabla-i b \mathbf{A}_{0}\right) u(x)\right|^{2} d x+\gamma \int_{\Gamma}|u(x)|^{2} d s(x) .
$$

\subsection{Main Result}

The operator $\mathcal{P}_{\gamma}^{b}$ has a compact resolvent, and thus its spectrum consists of an increasing sequence of eigenvalues. We are interested in examining the asymptotics of the principal eigenvalue

$$
\lambda_{1}(b, \gamma)=\inf _{u \in H^{1}(\Omega)} \frac{\mathcal{Q}_{\gamma}^{b}(u)}{\|u\|_{L^{2}(\Omega)}^{2}}
$$

when $b>0$ is fixed and the Robin parameter $\gamma$ tends to $-\infty$.

Theorem 1.1 Let $b>0$. Then, as $\gamma \rightarrow-\infty$,

$$
\lambda_{1}(b, \gamma)=-\gamma^{2}+\gamma+\inf _{m \in \mathbb{Z}}\left(m-\frac{b}{2}\right)^{2}-\frac{1}{2}+o(1) .
$$

The first two terms in the asymptotic expansion given in Theorem 1.1 are well known after many contributions (see [17-19] for the case $b=0$ and [13] for the case $b>0$ ); however, the third correction term is new for the disc geometry for $b>0$. The recent contribution [15, Thm. 1.5] shows that Theorem 1.1 continues to hold in the case $b=0$.

The proof of Theorem 1.1 can be easily modified to cover the situation of the annulus $\mathcal{A}_{\varrho}=\left\{x \in \mathbb{R}^{2}: \varrho<|x|<1\right\}$ (that is when take $\Omega=\mathcal{A}_{\varrho}$ and $\Gamma=\{|x|=$ $1\} \cap\{|x|=\varrho\}$ in (1.4)). The aymptotics for the principal eigenvalue in this case is the same as the one in Theorem 1.1. The reason is that the corresponding eigenfunctions are concentrated near the circle $\{|x|=1\}$. More details on this point are given in Remark 2.1

\subsection{Lack of Strong Diamagnetism}

The celebrated diamagnetic inequality yields

$$
\lambda_{1}(b, \gamma) \geqslant \lambda_{1}(\gamma, 0) .
$$

By using Theorem 1.1, we can quantify the diamagnetic inequality as follows

$$
\lambda_{1}(b, \gamma)-\lambda_{1}(0, \gamma) \underset{\gamma \rightarrow-\infty}{\sim} e(b):=\inf _{m \in \mathbb{Z}}\left(m-\frac{b}{2}\right)^{2} .
$$


Connected to the diamagnetic inequality is the property of strong diamagnetism [1]; this is whether the function $b \mapsto \lambda_{1}(b, \gamma)$ is monotone increasing on some interval $\left[b_{0},+\infty\right) \subset \mathbb{R}_{+}$.

As consequence of Theorem 1.1, we obtain a counterexample to strong diamagnetism.

Corollary 1.2 There exists $\gamma_{0}<0$ such that, for all $\gamma \in\left(-\infty, \gamma_{0}\right]$, the function $b \mapsto \lambda_{1}(b, \gamma)$ is not monotone increasing.

Besides its mathematical interest, the question of strong diamagnetism has applications to Physics, particularly in the context of superconductivity [2]. In the case of a simply connected domain subject to a uniform applied magnetic field and Neumann boundary condition $(\gamma=0)$, strong diamagnetism holds [3, 4]. Counter examples of strong diamagnetism exist for uniform magnetic fields in non-simply connected domains, or for non-uniform magnetic fields in simply connected domains [6,9]. Interestingly, the Robin boundary condition has the unique feature where strong diamagnetism fails for the disc (which is a simply connected domain) even when it is subject to a uniform applied magnetic field.

Corollary 1.2 results from the following statement. Given a positive real number $A$, there exist $\gamma_{0}<0$ and $A<b_{1}<b_{2}<b_{3}$ such that, for all $\gamma \in\left(-\infty, \gamma_{0}\right.$,

$$
\lambda_{1}\left(b_{1}, \gamma\right)<\lambda_{1}\left(b_{2}, \gamma\right) \& \lambda_{1}\left(b_{2}, \gamma\right)>\lambda_{1}\left(b_{3}, \gamma\right) .
$$

We can simply select the constants $b_{i}$ as follows

$$
b_{1}=2 n_{0}, \quad b_{2}=2 n_{0}+1, \quad b_{3}=2 n_{0}+\frac{3}{2},
$$

where $n_{0}$ is the smallest natural number satisfying $n_{0}>A$; the conclusion then follows from Theorem 1.1.

Using the periodicity of the function $b \mapsto e(b)$, given a natural number $N$, we can select $\gamma_{1}<0$ such that

$$
b_{1, i}:=b_{1}+i<b_{2, i}:=b_{2}+i<b_{3, i}:=b_{3}+i
$$

with the following two inequalities

$$
\lambda_{1}\left(b_{1, i}, \gamma\right)<\lambda_{1}\left(b_{2, i}, \gamma\right), \quad \lambda_{1}\left(b_{2, i}, \gamma\right)>\lambda_{1}\left(b_{3, i}, \gamma\right),
$$

holding for all $\gamma \leqslant \gamma_{1}$ and $i \in\{1,2, \cdots, N\}$ (Fig. 1).

\subsection{The Little-Parks Effect}

When cooled below a certain critical temperature, a normal conductor becomes a superconductor and looses electrical resistance. The Little-Parks experiment displays oscillations in the critical temperature of a superconductor as the applied magnetic field varies. Typically, the superconducting sample used in the experiment is a thin ring. From a mathematical perspective, the trivial normal solution of the GinzburgLandau equations changes back and forth from stable to unstable states.

Earlier contributions produced such an oscillatory behavior by topological obstructions (e.g. perforated domains $[6,9,11]$ ) or by the non-uniformity of the 


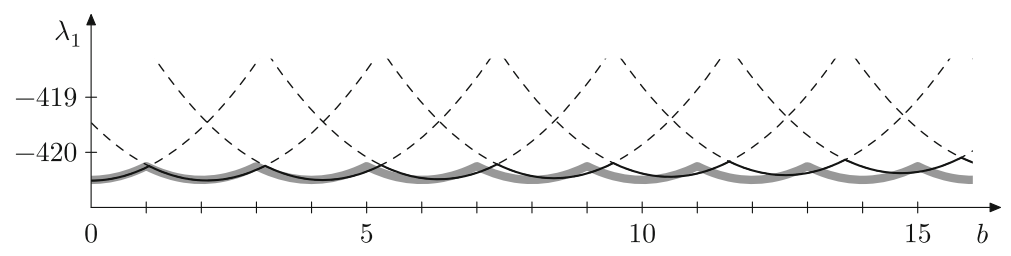

Fig. 1 We fix $\gamma=-20$. The solid black curve is the graph of the function $b \mapsto \lambda_{1}(b, \gamma)$ for $0<b<16$, calculated numerically using Wolfram Mathematica and the fact that the eigenvalues of the fiber operators (see Section 2.4) satisfy certain equations involving Whittaker functions. The dashed curves show how these eigenvalues continue outside the interval where they give the minimum energy. The gray thick curve in the background is the graph of the function $b \mapsto-\gamma^{2}+\gamma+\inf _{m \in \mathbb{Z}}(m-b / 2)^{2}-1 / 2$

magnetic field [6, 12]. The present contribution signals the role of the attractive Robin condition in producing normal-superconducting oscillations in the quite simple setting of a simply connected domain and uniform magnetic field.

Using a model for a superconductor with enhanced surface $[5,16]$, we can use Theorem 1.1 to estimate the critical temperature as a function of the applied magnetic field, consistent with the Little-Parks experiment. The novelty in our situation is that, unlike the Little-Parks experiment, the superconducting sample is a disc subject to a uniform magnetic field.

The model we study is a variant of the Ginzburg-Landau energy by adding a (negative) surface energy term (amounting for the enhanced surface). The surface term can be derived naturally starting from a Ginzburg-Landau model for two adjust superconductors [14, Thm. 1.2].

Following the presentation in [7, Sec. 3], we introduce the functional

$$
\begin{aligned}
& \mathcal{E}^{\text {phys }}(u, \mathbf{a})=\frac{\hbar^{2}}{2 m \ell} \int_{\partial \widetilde{\Omega}}|u|^{2} d \sigma(\tilde{x})+ \\
& \int_{\widetilde{\Omega}}\left(\frac{1}{2 m}\left|\left(\hbar \nabla-i \frac{2 e}{c} \mathbf{a}\right) u\right|^{2}+\alpha(T)|u|^{2}+\frac{\beta}{2}|u|^{4}+\frac{1}{8 \pi}|\operatorname{curl} \mathbf{a}-H|^{2}\right) d \tilde{x} .
\end{aligned}
$$

Here $(u, \mathbf{a})$ describes the superconducting properties $\left((u, \mathbf{a}) \equiv\left(0, H \mathbf{A}_{0}\right)\right.$ signifies the normal state, where $\mathbf{A}_{0}$ is introduced in (1.3)); $T$ denotes the temperature; $\hbar, e, c, m$, $\beta$ are positive constants; $H \geqslant 0$ measures the intensity of the applied magnetic field and $\ell<0$ models the enhanced surface. The disc $\widetilde{\Omega}=\left\{\tilde{x} \in \mathbb{R}^{2}:|\tilde{x}|<R\right\}$ is the horizontal cross section of the superconducting sample. The magnetic permeability $\mu_{0}$ in $\widetilde{\Omega}$ is assumed uniform, so we take $\mu_{0}=1$. For later use, we introduce the Ginzburg-Landau parameter

$$
\kappa=\sqrt{\frac{m^{2} \beta c^{2}}{8 \pi e^{2} \hbar}} .
$$

Among all the parameters in (1.7), only $\alpha(T)$ depends on the temperature $T$; consequently, $\kappa$ is temperature independent. The expression of $\alpha(T)$ is given via the 
following relation

$$
\alpha(T)=\frac{\hbar^{2}}{2 m \xi_{0}^{2}}\left(\frac{T}{T_{c_{0}}}-1\right),
$$

where $\xi_{0}>0$ is a temperature independent parameter, the coherence length at zero temperature. The parameter $T_{c_{0}}$ is the critical temperature of the superconductor occupying $\widetilde{\widetilde{\Omega}}$ in the absence of a magnetic field (i.e. when $H=0$ ).

We will express the functional in (1.7) in temperature independent units, and introduce some notation

$$
\begin{gathered}
\tilde{x}=R x, \tilde{\Omega}=R \Omega, u(\tilde{x})=\sqrt{\frac{|\alpha(0)|}{\beta}} \psi(R x), \\
\mathbf{a}(\tilde{x})=\frac{c \hbar}{2 e \xi_{0}^{2}} R \mathbf{A}(R x), b=\frac{c \hbar}{2 e \xi_{0}^{2}} R H, \gamma=\frac{R}{\ell} .
\end{gathered}
$$

The functional in (1.7) becomes

$$
\mathcal{E}^{\text {phys }}(u, \mathbf{a})=\frac{|\alpha(0)| \hbar^{2}}{2 m \beta} \mathcal{E}(\psi, \mathbf{A}),
$$

where

$$
\begin{aligned}
& \mathcal{E}(\psi, \mathbf{A})=\gamma \int_{\partial \Omega}|\psi|^{2} d \sigma(x)+ \\
& \int_{\Omega}\left(|(\nabla-i \mathbf{A}) \psi|^{2}-\frac{R^{2}}{\xi_{0}^{2}} \mu(T)|\psi|^{2}+\frac{R^{2}}{2 \xi_{0}^{2}}|\psi|^{4}+\kappa^{2}|\operatorname{curl} \mathbf{A}-b|^{2}\right) d x,
\end{aligned}
$$

and

$$
\mu(T):=1-\frac{T}{T_{c_{0}}} .
$$

The functional in (1.10) is defined on the space

$$
\mathcal{H}=H^{1}(\Omega ; \mathbb{C}) \times H^{1}\left(\Omega ; \mathbb{R}^{2}\right) .
$$

Clearly, the normal solution $\left(0, b \mathbf{A}_{0}\right)$ is a critical point of the functional in (1.10); it is said to be stable if it is a local minimizer. Using the direct method of the calculus of variations, we can prove that a minimizer $\left(\psi_{*}, \mathbf{A}_{*}\right) \in \mathcal{H}$ of $\mathcal{E}$ exists (cf. [7, Sec. 3]).

Recall the eigevalue $\lambda_{1}(b, \gamma)$ introduced in (1.5). Linearizing the functional in (1.10) near the normal state $\left(0, b \mathbf{A}_{0}\right)$, we get the following:

- If $\lambda_{1}(b, \gamma)<\frac{R^{2}}{\xi_{0}^{2}} \mu(T)$, then the normal state is not stable and the global minimizer $\left(\psi_{*}, \mathbf{A}_{*}\right)$ is non-trivial in the sense that $\psi_{*} \neq \equiv 0$;

- If $\lambda_{1}(b, \gamma)>\frac{R^{2}}{\xi_{0}^{2}} \mu(T)$, then the normal state is a local minimizer.

Consequently, we introduce the critical temperature $T_{c}(b)$, in the non-zero magnetic field $b$, as the solution of the equation

$$
\lambda_{1}(b, \gamma)=\frac{R^{2}}{\xi_{0}^{2}} \mu(T) \text {. }
$$


Thanks to (1.11) we find that

$$
T_{c}(b)=\left(1-\frac{\xi_{0}^{2}}{R^{2}} \lambda_{1}(b, \gamma)\right) T_{c_{0}}
$$

Using Theorem 1.1, we can estimate $T_{c}(b)$ as $\gamma \rightarrow-\infty$; we find

$$
T_{c}(b)=\left[1-\frac{\xi_{0}^{2}}{R^{2}}\left(-\gamma^{2}+\gamma+e(b)-\frac{1}{2}\right)\right] T_{c_{0}}+o(1),
$$

where $e(b)$ is introduced in (1.6). It is worth noticing that

$-T_{c}(b)>T_{c_{0}}$;

- Up to approximation errors, $T_{c}(b)$ is a periodic function of $b$, which is consistent with the Little-Parks effect;

- For $T<T_{c}(b)$, the global minimizer of $\mathcal{E}$ is non-trivial (in the sense $\psi_{*} \not \equiv 0$ ); while for $T>T_{c}(b)$, the normal solution is a local minimizer of $\mathcal{E}$.

\section{Proof of Theorem 1.1}

\subsection{Outline}

The proof consists of several reductions to operators that are easier to handle. In the first step we change parameter to have a semi-classical parameter. We then observe that we have localization close to the boundary, make a Fourier decomposition, and express the interesting operators and quadratic forms in suitable coordinates. Some effective operators appear, and we expand their eigenvalues in terms of the semiclassical parameter.

Since we are not looking at the large magnetic field limit, the potential that appears in polar coordinates is easier to handle since the angular momentum and magnetic field strength do not compete against each other.

\subsection{Translation of Theorem 1.1 into a Semi-Classical Statement}

It is convenient to work in a semi-classical framework. We do so by introducing the semi-classical parameter $h=\gamma^{-2}$. Then $h \rightarrow 0_{+}$when $\gamma \rightarrow-\infty$, and the quadratic form $\mathcal{Q}_{\gamma}^{b}$ can be written as

$$
\mathcal{Q}_{\gamma}^{b}(u)=h^{-2}\left(\int_{\Omega}\left|\left(h \nabla-i b h \mathbf{A}_{0}\right) u(x)\right|^{2} d x-h^{3 / 2} \int_{\partial \Omega}|u(x)|^{2} d s(x)\right) .
$$

Consequently, we get the $h$-dependent self-adjoint operator

$$
\mathcal{L}_{h}=-\left(h \nabla-i b h \mathbf{A}_{0}\right)^{2},
$$

with domain

$$
D\left(\mathcal{L}_{h}\right)=\left\{u \in H^{2}(\Omega): v \cdot\left(\nabla-i b \mathbf{A}_{0}\right) u-h^{1 / 2} u=0 \text { on } \partial \Omega\right\} .
$$


The spectra of the operators $\mathcal{P}_{\gamma}^{b}$ and $\mathcal{L}_{h}$ are related as

$$
\sigma\left(\mathcal{P}_{\gamma}^{b}\right)=h^{-2} \sigma\left(\mathcal{L}_{h}\right)
$$

Let $\mu_{1}(h, b)$ be the principal eigenvalue of the operator $\mathcal{L}_{h}$. Theorem 1.1 can be rephrased as follows.

Theorem 2.1 Let $b>0$. Then, as $h \rightarrow 0_{+}$,

$$
\mu_{1}(h, b)=-h-h^{3 / 2}+\left(\inf _{m \in \mathbb{Z}}\left(m-\frac{b}{2}\right)-\frac{1}{2}\right) h^{2}+o\left(h^{2}\right) .
$$

\subsection{Reduction to a Thin Ring}

Our aim is to work in (a variant of) polar coordinates. However, if we change directly to polar coordinates we will get some illusive problems at the origin with negative powers of $r=|x|$.

For small $h$, the ground states of the operator $\mathcal{L}_{h}$ are localized near the boundary of $\Omega$ (Proposition 2.3 below). This will allow us to work in an annulus instead of the disc. Before we give the localization result we show that there exists a sufficiently small eigenvalue for small $h$.

Lemma 2.2 Let $b>0$. Then there exists $h_{0} \in(0,1)$ such that for all $h \in\left(0, h_{0}\right)$

$$
\mu_{1}(h, b) \leqslant-h-\frac{1}{2} h^{3 / 2} .
$$

Proof Let $u(x)=c \exp \left(h^{-1 / 2}(|x|-1)\right)$, where $c$ is chosen so that $u$ becomes normalized in $L^{2}(\Omega)$. A direct calculation gives

$$
\frac{h^{2} \mathcal{Q}_{\gamma}^{b}(u)}{\|u\|^{2}}=-h-h^{3 / 2}+\mathcal{O}\left(h^{2}\right) .
$$

By changing the coefficient in front of $h^{3 / 2}$ we get the existence of $h_{0} \in(0,1)$ such that the claimed inequality holds for $h \in\left(0, h_{0}\right)$.

Proposition 2.3 (localization of ground states) Let $M \in(-1,0)$. For all $\alpha<\sqrt{-M}$, there exist constants $C>0$ and $h_{0} \in(0,1)$ such that, if $u_{h}$ is a normalized ground state of $\mathcal{L}_{h}$ with eigenvalue bounded above by $M h$, then, for all $h \in\left(0, h_{0}\right)$,

$$
\int_{\Omega}\left(\left|u_{h}(x)\right|^{2}+h\left|\left(\nabla-i b \mathbf{A}_{0}\right) u_{h}(x)\right|^{2}\right) \exp \left(\frac{2 \alpha \operatorname{dist}(x, \partial \Omega)}{h^{1 / 2}}\right) d x \leqslant C .
$$

The proof of Proposition 2.3 is similar to the one of [10, Thm. 5.1], and we leave out the details.

As a consequence of the concentration properties of the ground states, we can approximate the principal eigenvalue $\mu_{1}(h, b)$ by a ground state energy $\tilde{\mu}(h, b, \rho)$ that we describe next. 
Let $\rho \in\left(0, \frac{1}{2}\right)$ and consider the annulus $\Omega_{h}=\left\{x \in \mathbb{R}^{2}: 1-h^{\rho}<|x|<1\right\}$. We introduce the quadratic form

$$
q_{h}^{b, \rho}(u)=h^{2} \int_{\Omega_{h}}\left|\left(\nabla-i b \mathbf{A}_{0}\right) u(x)\right|^{2} d x-h^{3 / 2} \int_{|x|=1}|u(x)|^{2} d s
$$

defined on functions in $H^{1}\left(\Omega_{h}\right)$ with trace zero on the inner part of the boundary $\Gamma_{\text {in }}:=\left\{x \in \mathbb{R}^{2}:|x|=1-h^{\rho}\right\}$. This quadratic form is related to a self-adjoint operator with mixed boundary conditions. Its lowest eigenvalue $\tilde{\mu}_{1}(h, b, \rho)$ is given by

$$
\tilde{\mu}_{1}(h, b, \rho)=\inf \frac{q_{h}^{b, \rho}(u)}{\int_{\Omega_{h}}|u(x)|^{2} d x}
$$

where the infimum is taken over all $u$ in the domain of the quadratic form (i.e. $u \in$ $H^{1}\left(\Omega_{h}\right)$ with $u=0$ on $\left.\Gamma_{\text {in }}\right)$.

Lemma 2.4 Assume that $b>0$ and $\rho \in\left(0, \frac{1}{2}\right)$. Then there exists $h_{0} \in(0,1)$ such that, for all $h \in\left(0, h_{0}\right)$,

$$
\mu_{1}(h, b)=\tilde{\mu}_{1}(h, b, \rho)+\mathcal{O}\left(\exp \left(-h^{\rho-\frac{1}{2}}\right)\right) .
$$

Proof The inequality $\mu_{1}(h, b) \leqslant \tilde{\mu}_{1}(h, b, \rho)$ is not asymptotic. It follows by the Dirichlet bracketing. If $\tilde{u}$ is a function minimizing the quotient in (2.4), then we can extend it by zero inside the annulus. Inserting the new function into the quadratic form for $\mathcal{L}_{h}$, we find that $\mu_{1}(h, b) \leqslant \tilde{\mu}_{1}(h, b, \rho)$.

To get a bound in the opposite direction, we cut off (smoothly) the eigenfunction $u_{h}$ corresponding to $\mu_{1}(h, b)$, since it does not satisfy the correct boundary condition if $|x|=1-h^{\rho}$. More precisely, we use in (2.4) the trial state defined as follows

$$
v_{h}(x)=\chi\left(h^{-\rho}(1-|x|)\right) u_{h}(x)
$$

where $\chi \in C_{c}^{\infty}(\mathbb{R})$ is supported in $[-1,1]$ and equal to 1 on $\left[-\frac{1}{2}, \frac{1}{2}\right]$.

Thanks to Proposition 2.3 (with the choice $\alpha=\frac{1}{2}$ ) the error introduced is exponentially small.

In light of (2.5), we finish the proof of Theorem 2.1 once we prove that

$$
\tilde{\mu}(h, b, \rho)=-h-h^{3 / 2}+\left(\inf _{m \in \mathbb{Z}}\left(m-\frac{b}{2}\right)^{2}-\frac{1}{2}\right) h^{2}+o\left(h^{2}\right) .
$$

The $m$ in the right-hand side stands for the quantized angular momentum. Our next step is to make a Fourier expansion that will reduce our study to the study of an infinite family (parametrized by $m \in \mathbb{Z}$ ) of ordinary differential operators.

Remark 2.1 If we work in the annulus, $\mathcal{A}_{\varrho}=\left\{x \in \mathbb{R}^{2}: \varrho<|x|<1\right\}$, Lemma 2.4 continues to hold too. In fact, the ground states are localized near the points of maximal (oriented) curvature of the boundary of $\mathcal{A}_{h}[10,13,17]$. Here, the notion of the (oriented) curvature is as follows. For a circle $\{|x|=r\}$, it is $-\frac{1}{r}$ if measured from the exterior, and $\frac{1}{r}$ if measured from the interior. For the boundary of 
$\mathcal{A}_{\varrho}=\left\{x \in \mathbb{R}^{2}: \varrho<|x|<1\right\}$, the curvature of the outer boundary is 1 and that of the inner boundary is $-1 / \varrho$. So the eigenfunctions continue to concentrate near the circle $\{|x|=1\}$.

\subsection{Reduction to Fiber Operators}

We recall that $b>0$ and $\rho \in\left(0, \frac{1}{2}\right)$ are considered to be fixed constants. In polar coordinates $\left(x_{1}=r \cos \theta, x_{2}=r \sin \theta\right)$ the quadratic form $q_{h}^{b, \rho}$ reads

$$
h^{2}\left(\int_{0}^{2 \pi} \int_{1-h^{\rho}}^{1}\left(\left|\partial_{r} u\right|^{2}+\frac{1}{r^{2}}\left|\left(\partial_{\theta}-i \frac{b}{2} r^{2}\right) u\right|^{2}\right) r d r d \theta-h^{-1 / 2} \int_{0}^{2 \pi}|u|^{2} d \theta\right) .
$$

Next, we use the completeness of the orthonormal family $\left\{e^{i m \theta} / \sqrt{2 \pi}\right\}_{m \in \mathbb{Z}}$ in $L^{2}([0,2 \pi])$, and write

$$
u(r, \theta)=\sum_{m \in \mathbb{Z}} u_{m}(r) \frac{e^{i m \theta}}{\sqrt{2 \pi}}
$$

Here we assume that each $u_{m}$ belongs to $L^{2}\left(\left(1-h^{\rho}, 1\right), r d r\right)$. We are led to study the family of quadratic forms

$$
u_{m} \mapsto h^{2}\left(\int_{1-h^{\rho}}^{1}\left(\left|u_{m}^{\prime}(r)\right|^{2}+\frac{1}{r^{2}}\left|\left(m-\frac{b}{2} r^{2}\right) u_{m}(r)\right|^{2}\right) r d r-h^{-1 / 2}\left|u_{m}(1)\right|^{2}\right) .
$$

Since we have localization to the outer circle, it is convenient to work with the variable $\tau=h^{-1 / 2}(1-r)$, the scaled distance from $|x|=1$. We write $\tilde{u}_{m}(\tau)=u_{m}(r)$ and denote by

$$
\delta:=h^{\rho-\frac{1}{2}}
$$

the upper limit of $\tau$. This amounts to introduce the unitary transformation $\mathcal{U}: L^{2}\left(\left(1-h^{\rho}, 1\right) ; r d r\right) \rightarrow L^{2}\left((0, \delta) ;\left(1-h^{1 / 2} \tau\right) d \tau\right), \mathcal{U} u(\tau)=h^{1 / 4} u\left(1-h^{1 / 2} \tau\right)$, which yields

$$
\begin{aligned}
& h^{2}\left(\int_{1-h^{\rho}}^{1}\left(\left|u^{\prime}(r)\right|^{2}+\frac{1}{r^{2}}\left|\left(m-\frac{b}{2} r^{2}\right) u(r)\right|^{2}\right) r d r-h^{-1 / 2}|u(1)|^{2}\right)= \\
& h \int_{0}^{\delta}\left\{\left|(\mathcal{U} u)^{\prime}(\tau)\right|^{2}+h\left(1-h^{1 / 2} \tau\right)^{-2}\left|\left(m-\frac{b}{2}\left(1-h^{1 / 2} \tau\right)^{2}\right) \mathcal{U} u\right|^{2}\right\}\left(1-h^{1 / 2} \tau\right) d \tau \\
& -h|\mathcal{U} u(0)|^{2} .
\end{aligned}
$$

The relevant quadratic forms to study is

$$
\begin{array}{r}
\tilde{u}_{m} \mapsto \\
\int_{0}^{\delta}\left\{\left|\tilde{u}_{m}^{\prime}(\tau)\right|^{2}+h\left(1-h^{1 / 2} \tau\right)^{-2}\left|\left(m-\frac{b}{2}\left(1-h^{1 / 2} \tau\right)^{2}\right) \tilde{u}_{m}\right|^{2}\right\}\left(1-h^{1 / 2} \tau\right) d \tau \\
-\left|\tilde{u}_{m}(0)\right|^{2}
\end{array}
$$


The differential operator that corresponds to this quadratic form acts as

$$
\mathcal{H}_{m, h}^{b, \rho}=-\frac{d^{2}}{d \tau^{2}}+\frac{h^{1 / 2}}{1-h^{1 / 2} \tau} \frac{d}{d \tau}+\frac{h}{\left(1-h^{1 / 2} \tau\right)^{2}}\left(m-\frac{b}{2}\left(1-h^{1 / 2} \tau\right)^{2}\right)^{2} .
$$

With domain

$$
D\left(\mathcal{H}_{\beta, h}\right)=\left\{u \in H^{2}((0, \delta)): u^{\prime}(0)=-u(0) \quad \text { and } \quad u(\delta)=0\right\} .
$$

$\mathcal{H}_{m, h}^{b, \rho}$ becomes self-adjoint in the weighted space $L^{2}\left((0, \delta),\left(1-h^{1 / 2} \tau\right) d \tau\right)$. We denote the smallest eigenvalue of $\mathcal{H}_{m, h}^{b, \rho}$ by $\lambda_{1}\left(\mathcal{H}_{m, h}^{b, \rho}\right)$. From the completeness and orthogonality of the family $\left\{e^{i m \theta}\right\}_{m \in \mathbb{Z}}$ it follows that

$$
\tilde{\mu}(h, b, \rho)=h \inf _{m \in \mathbb{Z}} \lambda_{1}\left(\mathcal{H}_{m, h}^{b, \rho}\right) .
$$

To take advantage of this equality we need information about $\lambda_{1}\left(\mathcal{H}_{m, h}^{b, \rho}\right)$. We will get the information needed by comparing with simpler operators. In fact, we will first compare with the weighted Laplace obtained by ignoring the third term in the right-hand side of (2.8). To do this, we first look at the simpler operator obtained by ignoring also the second term.

\subsection{A 1D Laplacian}

The spectrum of the operator $-\frac{d^{2}}{d \tau^{2}}$ in $L^{2}\left(\mathbb{R}_{+}\right)$with domain $\mathcal{D}_{0}=\left\{u \in H^{2}\left(\mathbb{R}_{+}\right)\right.$: $\left.u^{\prime}(0)=-u(0)\right\}$ is explicitly known (see [10]). It consists of the simple eigenvalue -1 together with the interval $[0,+\infty)$. A normalized eigenfunction corresponding to the eigenvalue -1 is given by

$$
u_{0}(\tau)=\sqrt{2} \exp (-\tau) .
$$

By the Fredholm alternative, we can invert the operator $-\frac{d^{2}}{d \tau^{2}}+1$ on the orthogonal complement of the eigenfunction $u_{0}$, and obtain functions in $\mathcal{D}_{0}$. The inverse can be expressed explicitly by solving a simple second order differential equation. That will be used later in Lemma 2.5 below.

\subsection{A Weighted 1D Laplacian}

Let $\rho \in\left(\frac{1}{4}, \frac{1}{2}\right)$ be a fixed constant. In the sequel, the parameter $h \in(0,1)$ varies so that $h^{\frac{1}{2}-\rho}<\frac{1}{3}$. We recall that $\delta=h^{\rho-\frac{1}{2}}$ and note that $\delta \rightarrow+\infty$ when $h \rightarrow 0_{+}$.

In the weighted space $L^{2}\left((0, \delta) ;\left(1-h^{1 / 2} \tau\right) d \tau\right)$, we introduce the self-adjoint operator,

$$
\mathcal{H}_{h}=-\frac{d^{2}}{d \tau^{2}}+\frac{h^{1 / 2}}{1-h^{1 / 2} \tau} \frac{d}{d \tau}
$$

with domain

$$
D\left(\mathcal{H}_{h}\right)=\left\{u \in H^{2}((0, \delta)): u^{\prime}(0)=-u(0) \quad \text { and } \quad u(\delta)=0\right\} .
$$


The operator $\mathcal{H}_{h}$ is defined starting from the closed quadratic form

$$
q_{h}(u)=\int_{0}^{\delta}\left|u^{\prime}(\tau)\right|^{2}\left(1-h^{1 / 2} \tau\right) d \tau-|u(0)|^{2},
$$

with domain ${ }^{1} \mathcal{D}\left(q_{h}\right)=\left\{u \in H^{1}(0, \delta): u(\delta)=0\right\}$.

The increasing sequence of the eigenvalues of $\mathcal{H}_{h}$ (counting multiplicities) is denoted by $\left(\lambda_{n}\left(\mathcal{H}_{h}\right)\right)_{n \in \mathbb{N}}$. In [10, Lem. $4.4 \&$ Prop. 4.5$]$ it is proved that

$$
\lambda_{1}\left(\mathcal{H}_{h}\right)=-1-h^{1 / 2}+o\left(h^{1 / 2}\right) \quad \text { and } \quad \lambda_{2}\left(\mathcal{H}_{h}\right) \geqslant \mathcal{O}\left(h^{\rho}\right) \quad\left(h \rightarrow 0_{+}\right) .
$$

We are going to refine the expansion of $\lambda_{1}\left(\mathcal{H}_{h}\right)$ by determining the term of order $h$.

Lemma 2.5 Assume that $\rho \in\left(\frac{1}{4}, \frac{1}{2}\right)$. Then, as $h \rightarrow 0_{+}$,

$$
\lambda_{1}\left(\mathcal{H}_{h}\right)=-1-h^{1 / 2}-\frac{1}{2} h+o(h) .
$$

Proof According to (2.14) there is a spectral gap of constant order, so it suffices to construct a trial state that will give an energy estimate of sufficient accuracy. We will construct a formal eigenfunction $u_{0}+h^{1 / 2} u_{1}+h u$ with a corresponding formal eigenvalue $\mu_{0}+h^{1 / 2} \mu_{1}+h \mu_{2}$.

To do this we expand the operator $\mathcal{H}_{h}$ formally in $h$ as

$$
\mathcal{H}_{h}=-\frac{d^{2}}{d \tau^{2}}+h^{1 / 2} \frac{d}{d \tau}+h \tau \frac{d}{d \tau}+\mathcal{O}\left(h^{\frac{1}{2}+2 \rho}\right) \frac{d}{d \tau},
$$

and note that, for $\rho \in\left(\frac{1}{4}, \frac{1}{2}\right), h^{\frac{1}{2}+2 \rho}=o(h)$ as $h \rightarrow 0_{+}$.

We work on the half-line $\mathbb{R}_{+}$, with the weight free operator in Section 2.5, and construct functions $u_{0}, u_{1}, u_{2}$ and coefficients $\mu_{0}, \mu_{1}, \mu_{2}$ such that

$$
\begin{aligned}
\left(-\frac{d^{2}}{d \tau^{2}}-\mu_{0}\right) u_{0} & =0, \\
\left(-\frac{d^{2}}{d \tau^{2}}-\mu_{0}\right) u_{1} & =-u_{0}^{\prime}+\mu_{1} u_{0}, \\
\left(-\frac{d^{2}}{d \tau^{2}}-\mu_{0}\right) u_{2} & =-u_{1}^{\prime}+\mu_{1} u_{1}-\tau u_{0}^{\prime}+\mu_{2} u_{0}, \\
\text { and } u_{i}^{\prime}(0) & =-u_{i}(0) \text { for } i \in\{0,1,2\} .
\end{aligned}
$$

The natural choice is then to choose $u_{0}$ the eigenfunction in (2.11) and $\mu_{0}$ the corresponding eigenvalue. Then we choose $\mu_{1}$ so that $-u_{0}^{\prime}+\mu_{1} u_{0}$ is orthogonal to $u_{0}$; after that we can determine $u_{1}$ since, by Fredholm's alternative, we can invert the operator $-\frac{d^{2}}{d \tau^{2}}-\mu_{0}$ on the orthogonal complement of $u_{0}$. Finally, we select $\mu_{2}$ so

\footnotetext{
${ }^{1}$ Recall that we consider $h \in(0,1)$ so that $h^{\frac{1}{2}} \delta=h^{\frac{1}{2}-\rho}<\frac{1}{3}$, which ensures that $\frac{2}{3} \leqslant 1-h^{1 / 2} \tau<1$ when $0<\tau<\delta$.
} 
that $-u_{1}^{\prime}+\mu_{1} u_{1}-\tau u_{0}^{\prime}+\mu_{2} u_{0}$ is othogonal to $u_{0}$ which allows us eventually to determine $u_{2}$. In that way we obtain

$$
\begin{gathered}
\mu_{0}=-1, \quad u_{0}(\tau)=\sqrt{2} \exp (-\tau), \\
\mu_{1}=-1, \quad u_{1}(\tau)=0 \\
\mu_{2}=-\frac{1}{2}, \quad u_{2}(\tau)=\left(-\frac{d^{2}}{d \tau^{2}}+1\right)^{-1}\left[\left(\tau-\frac{1}{2}\right) u_{0}(\tau)\right]=\left(\frac{\tau^{2}}{4}-\frac{1}{8}\right) u_{0}(\tau) .
\end{gathered}
$$

Now, consider the function,

$$
f(\tau)=\chi\left(\frac{\tau}{\delta}\right)\left(u_{0}(\tau)+h^{1 / 2} u_{1}(\tau)+h u_{2}(\tau)\right),
$$

where $\chi \in C_{c}^{\infty}([0, \infty))$ satisfies,

$$
0 \leqslant \chi \leqslant 1 \text { in }[0, \infty), \quad \chi=1 \text { in }[0,1 / 2) \text { and } \chi=0 \text { in }[1,+\infty) \text {. }
$$

The function $f$ is in the domain of the operator $\mathcal{H}_{h}$, and by construction it is almost normalized in the weighted Hilbert space, having a norm of size $1+\mathcal{O}(h)$. Moreover, a straightforward estimate shows that

$$
\left\|\left\{\mathcal{H}_{h}-\left(\mu_{0}+\mu_{1} h^{1 / 2}+\mu_{2} h\right)\right\} f\right\|_{L^{2}\left((0, \delta) ;\left(1-h^{1 / 2} \tau\right) d \tau\right)}=o(h) .
$$

The spectral theorem and (2.14) now completes the proof of Lemma 2.5.

\subsection{Reducing the angular momentum}

We disqualify some values of the angular momentum $m$ from minimizing the righthand side in (2.10).

Proposition 2.6 Assume that $b>0, \rho \in\left(\frac{1}{4}, \frac{1}{2}\right)$ and that $h \in(0,1)$. If $|m|>$ $(1+\sqrt{2}) b / 2$, then

$$
\lambda_{1}\left(\mathcal{H}_{m, h}^{b, \rho}\right)>\inf _{\ell \in \mathbb{Z}} \lambda_{1}\left(\mathcal{H}_{\ell, h}^{b, \rho}\right)
$$

Proof We note that if $m=0$, then we have the bound

$$
\frac{h}{\left(1-h^{1 / 2} \tau\right)^{2}}\left(m-\frac{b}{2}\left(1-h^{1 / 2} \tau\right)^{2}\right)^{2} \leqslant \frac{b^{2}}{4} h
$$

on the potential term in $\mathcal{H}_{m, h}^{b, \rho}$. Comparing quadratic forms,

$$
\inf _{\ell \in \mathbb{Z}} \lambda_{1}\left(\mathcal{H}_{\ell, h}^{b, \rho}\right) \leqslant \lambda_{1}\left(\mathcal{H}_{0, h}^{b, \rho}\right) \leqslant \lambda_{1}\left(\mathcal{H}_{h}\right)+\frac{b^{2}}{4} h
$$

where $\lambda_{1}\left(\mathcal{H}_{h}\right)$ is the lowest eigenvalue of the operator introduced in (2.12).

We expand the square and estimate the potential term again, using the fact that $1-h^{\rho}<1-h^{1 / 2} \tau<1$

$$
\begin{aligned}
\frac{1}{\left(1-h^{1 / 2} \tau\right)^{2}}\left(m-\frac{b}{2}\left(1-h^{1 / 2} \tau\right)^{2}\right)^{2} & =\frac{m^{2}}{\left(1-h^{1 / 2} \tau\right)^{2}}-m b+\frac{b^{2}}{4}\left(1-h^{1 / 2} \tau\right)^{2} \\
& \geqslant m^{2}-m b=\left(m-\frac{b}{2}\right)^{2}-\frac{b^{2}}{4} .
\end{aligned}
$$


We compare the quadratic forms and invoke (2.15) to find that

$$
\begin{aligned}
\lambda_{1}\left(\mathcal{H}_{m, h}^{b, \rho}\right) & \geqslant \lambda_{1}\left(\mathcal{H}_{h}\right)+h\left(\left(m-\frac{b}{2}\right)^{2}-\frac{b^{2}}{4}\right) \\
& \geqslant \inf _{\ell \in \mathbb{Z}} \lambda_{1}\left(\mathcal{H}_{\ell, h}^{b, \rho}\right)+h\left(\left(m-\frac{b}{2}\right)^{2}-\frac{b^{2}}{2}\right) .
\end{aligned}
$$

If $|m|>(1+\sqrt{2}) b / 2$ then $(m-b / 2)^{2}>b^{2} / 2$ and thus

$$
\lambda_{1}\left(\mathcal{H}_{m, h}^{b}\right)>\inf _{\ell \in \mathbb{Z}} \lambda_{1}\left(\mathcal{H}_{\ell, h}^{b, \rho}\right) .
$$

\subsection{A Family of 1D Operators}

Assume that $A>0, \rho \in\left(0, \frac{1}{2}\right)$ and $b>0$ are fixed constants. Suppose that the parameters $h \in(0,1)$ and $m \in \mathbb{Z}$ vary as follows

$$
h^{\frac{1}{2}-\rho}<\frac{1}{3} \text { and }|m| \leqslant A .
$$

We introduce also the following ground state energy

$$
\hat{\lambda}(b, A)=\inf _{\substack{m \in \mathbb{Z} \\|m| \leqslant A}} \lambda_{1}\left(\mathcal{H}_{m, h}^{b}\right) .
$$

Proposition 2.7 Given $A, b>0$ and $\rho \in\left(\frac{1}{4}, \frac{1}{2}\right)$, it holds that

$$
\hat{\lambda}(b, A)=-1+h^{1 / 2}+\left(\hat{\beta}(b, A)-\frac{1}{2}\right) h+o(h),
$$

where

$$
\hat{\beta}(b, A)=\inf _{\substack{m \in \mathbb{Z} \\|m| \leqslant A}}\left(m-\frac{b}{2}\right)^{2} .
$$

Remark 2.2 Given $b>0$, there exists $A_{0}>0$ such that, for all $A \geqslant A_{0}$,

$$
\hat{\beta}(b, A)=\inf _{m \in \mathbb{Z}}\left(m-\frac{b}{2}\right)^{2} .
$$

Proof We will write estimates that hold uniformly with respect to $(m, h)$ obeying the conditions in (2.16). A calculation shows that

$$
\begin{aligned}
& \frac{1}{\left(1-h^{1 / 2} \tau\right)^{2}}\left(m-\frac{b}{2}\left(1-h^{1 / 2} \tau\right)^{2}\right)^{2}-\left(m-\frac{b}{2}\right)^{2} \\
& =\left(2-h^{1 / 2} \tau\right)\left(\frac{1}{\left(1-h^{1 / 2} \tau\right)^{2}} m^{2}-\frac{b^{2}}{4}\right) h^{1 / 2} \tau .
\end{aligned}
$$

Thus, using that $0 \leqslant h^{1 / 2} \tau \leqslant h^{\rho}$ for $\tau \in(0, \delta)$, we can write

$$
\left|\frac{1}{\left(1-h^{1 / 2} \tau\right)^{2}}\left(m-\frac{b}{2}\left(1-h^{1 / 2} \tau\right)^{2}\right)^{2}-\left(m-\frac{b}{2}\right)^{2}\right| \leqslant C h^{\rho},
$$


where $C$ is a constant independent from $m \in[-A, A]$. Consequently, the min-max principle yields

$$
\left|\lambda_{1}\left(\mathcal{H}_{m, h}^{b, \rho}\right)-\left(\lambda_{1}\left(\mathcal{H}_{h}\right)+\left(m-\frac{b}{2}\right)^{2} h\right)\right| \leqslant C h^{1+\rho},
$$

where $\mathcal{H}_{h}$ is the operator introduced in (2.12). Now, using Lemma 2.5, we finish the proof of Proposition 2.7.

\subsection{End of Proof}

We now have everything we need to finish the proof of Theorem 1.1. Remember that $b>0$ was given in the theorem. We start by choosing some $\rho \in\left(\frac{1}{4}, \frac{1}{2}\right)$. Then, by combining the Propositions 2.6 and 2.7 (see also Remark 2.2) with Lemma 2.5 we find that, as $h \rightarrow 0_{+}$,

$$
\inf _{m \in \mathbb{Z}} \lambda_{1}\left(\mathcal{H}_{m, h}^{b, \rho}\right)=-1-h^{1 / 2}+\left(\inf _{m \in \mathbb{Z}}\left(m-\frac{b}{2}\right)-\frac{1}{2}\right) h+o(h) .
$$

From (2.10) we now conclude that, as $h \rightarrow 0_{+}$,

$$
\tilde{\mu}(h, b, \rho)=-h-h^{3 / 2}+\left(\inf _{m \in \mathbb{Z}}\left(m-\frac{b}{2}\right)-\frac{1}{2}\right) h^{2}+o\left(h^{2}\right) .
$$

As we mentioned in the end of Section 2.3 this was sufficient to prove Theorem 2.1 which in turn was a reformulation of Theorem 1.1.

Acknowledgments This work started when A. Kachmar visited the mathematics department in Lund University. The research of A. Kachmar is supported by a grant from the Lebanese university within the project "Analytical and numerical aspects of the Ginzburg-Landau model". The authors thank the anonymous referee for valuable comments and suggestions.

Funding Information Open access funding provided by Lund University.

Open Access This article is licensed under a Creative Commons Attribution 4.0 International License, which permits use, sharing, adaptation, distribution and reproduction in any medium or format, as long as you give appropriate credit to the original author(s) and the source, provide a link to the Creative Commons licence, and indicate if changes were made. The images or other third party material in this article are included in the article's Creative Commons licence, unless indicated otherwise in a credit line to the material. If material is not included in the article's Creative Commons licence and your intended use is not permitted by statutory regulation or exceeds the permitted use, you will need to obtain permission directly from the copyright holder. To view a copy of this licence, visit http://creativecommonshorg/licenses/by/4.0/.

\section{References}

1. Erdös, L.: Dia- and paramagnetism for nonhomogeneous magnetic fields. J. Math Phys. 38(3), 1289_ 1317 (1997)

2. Fournais, S., Helffer, B.: Spectral Methods in Surface Superconductivity. Progress in Nonlinear Differential Equations and Their Applications, vol. 77. Birkhäuser (2010)

3. Fournais, S., Helffer, B.: On the third critical field in Ginzburg- Landau theory. Comm. Math. Phys. 266(1), 153-196 (2006)

4. Fournais, S., Helffer, B.: Strong diamagnetism for general domains and applications. Ann. Inst. Fourier 57(7), 2389-2400 (2007) 
5. Fink, H.K., Joiner, W.C.H.: Surface nucleation and boundary conditions in superconductors. Phys. Rev Lett. 23, 120-123 (1969)

6. Fournais, S., Persson-Sundqvist, M.: Lack of diamagnetism and the Little-Parks effect. Comm. M.th. Phys. 337(1), 191-224 (2015)

7. Giorgi, T., Smits, R.: Eigenvalue estimates and critical temperature in zero fields for enhanced surface superconductivity. Z. Angew. Math Phys. 57, 1-22 (2006)

8. Helffer, B.: Spectral Theory and Its Applications. Cambridge Studies in Advanced Mathematics, vol. 139 (2013)

9. Helffer, B., Kachmar, A.: Thin domain limit and counterexamples to strong diamagnetism. arXiv: 1905.06152

10. Helffer, B., Kachmar, A.: Eigenvalues for the Robin Laplacian in domains with variable curvature. Trans. A.er. Math. Soc. 369(5), 3253-3287 (2017)

11. Kachmar, A., Pan, X.B.: Superconductivity and the Aharonov-Bohm effect. C. R Math. 357(2), 216220 (2019)

12. Kachmar, A., Pan, X.B.: Oscillatory patterns in the Ginzburg-Landau model driven by the AharonovBohm potential. Preprint

13. Kachmar, A.: Diamagnetism versus Robin condition and concentration of ground states. Asymptot. Anal. 98(4), 341-375 (2016)

14. Kachmar, A.: Magnetic vortices for a Ginzburg-Landau type energy with discontinuous constrain. ESAIM: COCV 16, 545-580 (2010)

15. Khalile, M., Ourmières-Bonafos, T., Pankrashkin, K.: Effective operators for Robin eigenvalues in domains with corners. Ann. Institut Fourier (in press)

16. Montevecchi, E., Indekeu, J.O.: Effects of confinement and surface enhancement on superconductivity. Phys. Rev. B. 62, 14359-14372 (2000)

17. Pankrashkin, K.: On the asymptotics of the principal eigenvalue problem for a Robin problem with a large parameter in a planar domain. Nanosystems: Phys. Chem. Math. 4(4), 474-483 (2013)

18. Pankrashkin, K., Popoff, N.: Mean curvature bounds and eigenvalues of Robin Laplacians. Calc. Var. Partial Differ. Equ. 54(2), 1947-1961 (2015)

19. Pankrashkin, K., Popoff, N.: An effective Hamiltonian for the eigenvalues asymptotics of a Robin Laplacian with a large parameter. J. Math. Pures et Appl. 106(4), 615-650 (2016)

Publisher's Note Springer Nature remains neutral with regard to jurisdictional claims in published maps and institutional affiliations. 